\title{
A formalization of one of the main claims of "The FAIR Guiding Principles for scientific data management and stewardship" by Wilkinson et al. $2016^{1}$
}

\author{
Michel Dumontier \\ Maastricht University, The Netherlands \\ E-mails: michel.dumontier@maastrichtuniversity.nl,michel.dumontier@gmail.com; ORCID: \\ https://orcid.org/0000-0003-4727-9435 \\ Editor: Cristina-Iulia Bucur (https://orcid.org/0000-0002-7114-6459) \\ Review comments from: Tobias Kuhn (https://orcid.org/0000-0002-1267-0234); Ricardo Usbeck \\ (https://orcid.org/0000-0002-0191-7211); Margherita Martorana (https://orcid.org/0000-0001-8004-0464); Cristina-Iulia \\ Bucur (https://orcid.org/0000-0002-7114-6459)
}

Received 25 June 2021

Accepted 17 November 2021

Abstract. Wilkinson et al. claimed in previous work that Adherence of a dataset to the FAIR Guiding Principles enables its automated discovery. We present here a formalization of that claim, stating that all things of class "adherence to the FAIR Guiding principles" that are in the context of a thing of class "data set" can generally have a relation of type "enables" to a thing of class "automated discovery" in the same context.

Keywords: Data set, adherence to the FAIR guiding principles, automated discovery

\section{Introduction}

Wilkinson et al. state that "the FAIR Principles put specific emphasis on enhancing the ability of machines to automatically find and use the data" [2]. We present here a formalization of the main scientific claim from this quote by using a semantic template called the super-pattern [1].

\section{Formalization}

Our formalization looks as follows:

\footnotetext{
${ }^{1}$ As RDF/nanopublication: http://purl.org/np/RA22JAQihYeiJkNIjvwnxLPmjuG74yPcRXpPyVX8DV6fA
} 


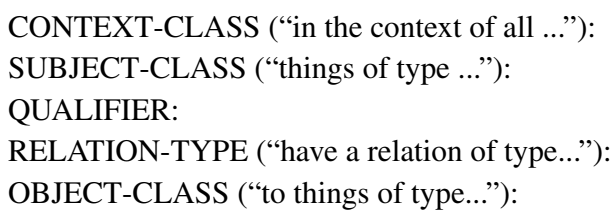

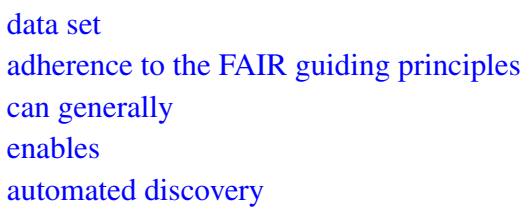

In the context class we use the "data set" (Q1172284) class from Wikidata. In subject class, we use a new minted class "adherence to the FAIR guiding principles" that is a subclass of "adherence" (Q85315455) and is related to the class "FAIR data principles" (Q29032644) from Wikidata. In the object class we minted a new class "automated discovery" that is a subclass of "discovery" (Q12772819) and is related to the class "automation" (Q184199) from Wikidata.

\section{RDF code}

This is our formalization as a nanopublication in TriG format:

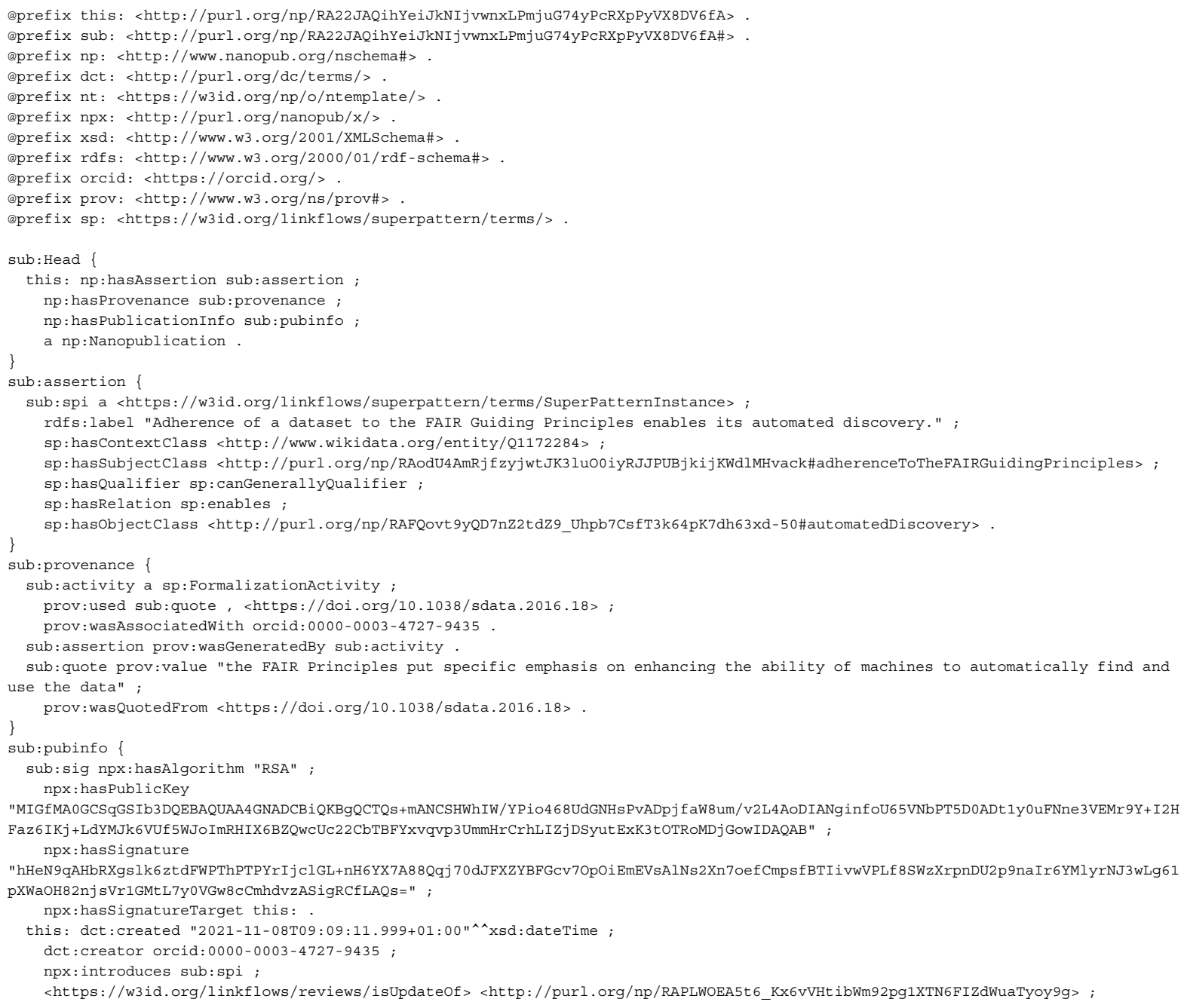


ht: wasCreatedFromProvenanceTemplate <http://purl.org/np/RAE1wniOy0yO39PlK9QkQ-wqbC3q-R2nXraP5huu8W39k> ; $\mathrm{nt}$ : wasCreatedFromPubinfoTemplate <http://purl.org/np/RAA2MfqdBCzmz9YVWj KLXNbyfBNCWSMmOqCNUxkk1maIM>,

<http://purl.org/np/RAOGu9Lh0BD4tbIRB9RG6RGRA_ObDh75NTbIqaWgxxs8M>;
nt: wasCreatedFromTemplate <http://purl.org/np/RAv68imZrEjfcp2rnEg1hzoBqEVC0cQMtp9_1Za0BxNM4> .

The following nanopublications introduce the newly minted classes in TriG format.

This is the class definition of "adherence to the FAIR guiding principles":

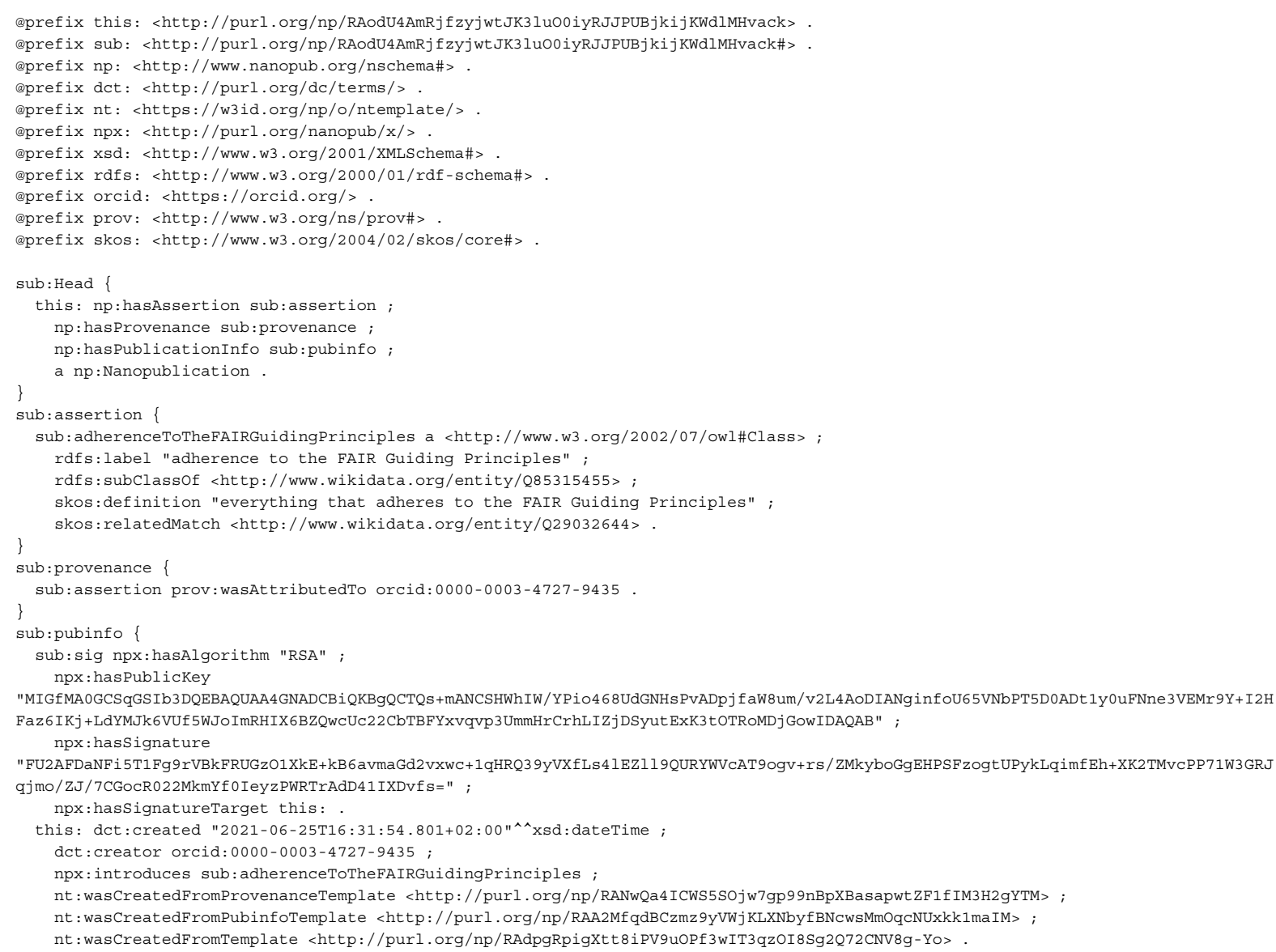

This is the class definition of "automated discovery":

@prefix this: <http://purl.org/np/RAFQovt9yQD7nZ2tdZ9_Uhpb7CsfT3k64pK7dh63xd-50>. @prefix sub: <http://purl.org/np/RAFQovt9yQD7nZ2tdZ9_Uhpb7CsfT3k64pK7dh63xd-50\#>

@prefix np: <http://www. nanopub.org/nschema\#>

@prefix dct: <http://purl.org/dc/terms/>

@prefix nt: <https://w3id.org/np/o/ntemplate/>

@prefix npx: <http://purl.org/nanopub/x/>

@prefix xsd: <http://www.w3.org/2001/XMLSchema\#>

@prefix rdfs: <http://www.w3.org/2000/01/rdf-schema\#>.

@prefix orcid: <https://orcid.org/>

@prefix prov: <http://www.w3.org/ns/prov\#>

@prefix skos: <http://www.w3.org/2004/02/skos/core\#>

sub:Head \{

this: np:hasAssertion sub:assertion

np: hasProvenance sub:provenance ;

$\mathrm{np}$ : hasPublicationInfo sub:pubinfo

a np:Nanopublication 


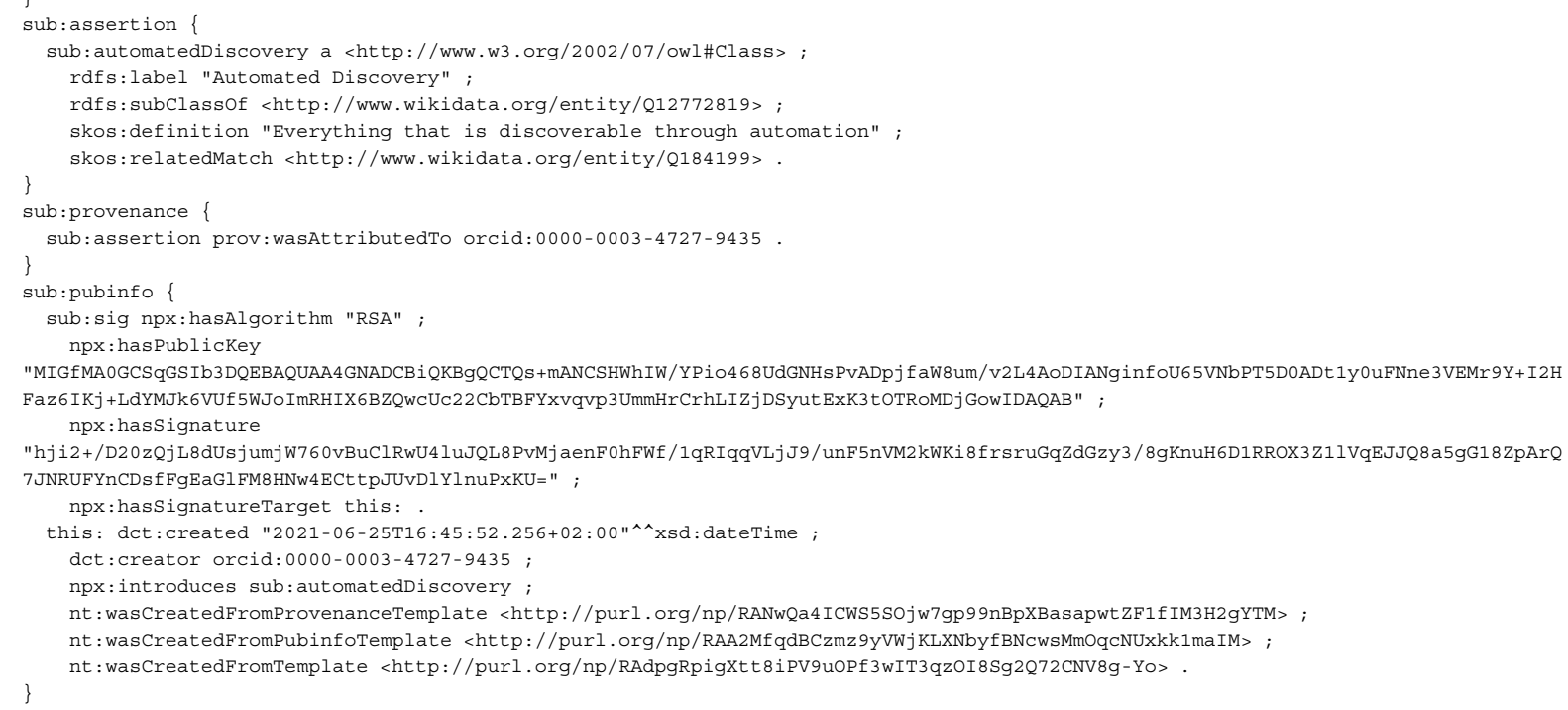

"MIGFMA0GCSqGSIb3DQEBAQUAA4GNADCBiQKBgQCTQS+mANCSHWhIW/YPiO468UdGNHs PvADpj faW8um/v2L4AoDIANginfoU65VNbPT5D0ADt1 Y0uFNne3VEMr9Y+I2H Faz6IKj+LdYMJk6VUf5WJoImRHIX6BZQWCUc22 CbTBFYxvqvp3UmmHrCrhLIZjDSyutExK3tOTRoMDjGowIDAQAB" ;

\section{References}

[1] C.I. Bucur, T. Kuhn, D. Ceolin and J. van Ossenbruggen, Expressing high-level scientific claims with formal semantics, in: Proceedings of the 11th Knowledge Capture Conference, 2021. doi:10.1145/3460210.3493561.

[2] M. Wilkinson, M. Dumontier, I. Aalbersberg et al., The FAIR Guiding Principles for scientific data management and stewardship, Sci Data 3 (2016), 160018. doi:10.1038/sdata.2016.18. 